\title{
Fast and efficient analysis of transient nonlinear heat conduction problems using combined approximations (CA) method
}

\author{
S. Z. Feng ${ }^{1}$, X. Y. Cui ${ }^{2, *}$, A. M. Li $^{1}$ \\ ${ }^{1}$ School of Mechatronic Engineering, China University of Mining and Technology, Xuzhou, 221116, \\ P. R. China \\ ${ }^{2}$ State Key Lab. of Advanced Design and Manufacturing for Vehicle Body, Hunan University, \\ Changsha, 410082, P. R. China
}

* Corresponding author. Tel: +86 7318821717 ; fax: +86 7318822051

E-mail address: szfenghnu@,126.com (X. Y. Cui). 


\title{
Fast and efficient analysis of transient nonlinear heat conduction problems using combined approximations (CA) method
}

\begin{abstract}
The combined approximations (CA) method is very efficient and can provide high quality results, when dealing with structural optimization problems. In this study, the CA method is utilized to reduce the computational effort in transient nonlinear heat transfer analysis. In the CA approach, binomial series are used as high quality basis vectors for reduced basis expression. Transient nonlinear temperature fields are then calculated without solving complete set of nonlinear system equations. Both two-dimensional and three-dimensional numerical problems are studied to verify the accuracy and efficiency of CA method, when solving transient nonlinear heat conduction problems.
\end{abstract}

Keywords: Transient heat transfer; Nonlinear; Combined approximations

\section{Introduction}

The transient nonlinear heat transfer analysis is of great importance in many practical engineering areas [1-3]. So much research has been done in recent years, focusing on solving such problems [4-6]. Since it is usually very difficult to find analytical solutions, numerical methods are widely employed for the analysis, such as finite element method (FEM) [7-8], meshless method [9-15], boundary element method (BEM) [16] and so on. The transient heat conduction process is time-dependent and hence, ordinary differential equations need to be solved in the time domain. Among 
existing methods that can be used for such analysis, the time difference method has become one of the most popular methods, which includes the forward, central and backward difference methods.

It is quite clear that the transient heat conduction analysis will involve extensive computational effort, especially when the time step is small or the scale of problem is large. This is mainly due to the fact that numerous system equations should be solved within each time step. In order to improve the efficiency, fast and reliable computational method is necessary for such analysis. In early $1990 \mathrm{~s}$, the research group of Kirsch published the first study on Combined Approximations (CA) method for structural optimization problems, in which massive system equations need to be solved repeatedly [17-19]. The CA method is used to evaluate structural response for changes of structures without solving complete set of modified system equations. The original responses of structures are usually utilized in the solution procedure and terms of binomial series are employed as high quality basis vectors in the reduced basis expression. Efficient local approximations and accurate global approximations are then combined to achieve effective solution procedures. Generally, the computational effort of CA method is much smaller than that of complete analysis. Since then, the effectiveness of CA method has been fully demonstrated, when dealing with structural optimization problems [20-26]. Accurate results and significant savings in computational effort can be achieved by this method, even when the changes of design variables are large. This method has been successfully used for a wide range of structural optimization problems [27-32]. 
In the transient nonlinear heat transfer process, thermal conductivity is not a constant due to the change of temperature. Therefore, the global system conduction matrix will vary with time and such a process is quite similar to the structural optimization process, in which global system stiffness matrix varies due to the change of design variables. So, the main objective of this study is to deal with the transient nonlinear heat conduction analysis more efficiently by taking advantage of CA approach. Transient temperature fields are calculated using combined approximations without solving complete set of nonlinear system equations. Both two-dimensional and three-dimensional numerical problems are investigated to study the accuracy and efficiency of CA method for transient nonlinear heat transfer analysis.

\section{Thermal governing equations and boundary conditions}

The differential equation governing transient heat conduction is given as

$$
\left(k_{x} \frac{\partial^{2} T}{\partial x^{2}}+k_{y} \frac{\partial^{2} T}{\partial y^{2}}+k_{z} \frac{\partial^{2} T}{\partial z^{2}}\right)+q_{v}=\rho c \frac{\partial T}{\partial t}
$$

where $T$ is the temperature, $q_{v}$ is the internal heat generation, $\rho$ is the density, $c$ is the specific heat, $k_{x}, k_{y}$ and $k_{z}$ are the thermal conductivities in the $x, y, z$ directions, respectively. Thermal boundary conditions are expressed as

Dirichlet boundary : $\left.T\right|_{\Gamma}=T_{k}$

Neumann boundary : $-\left.k \frac{\partial T}{\partial n_{0}}\right|_{\Gamma}=q_{0}$

Robin boundary : $-\left.k \frac{\partial T}{\partial n_{0}}\right|_{\Gamma}=h_{c}\left(T-T_{\infty}\right)$

Adiabatic boundary : $-\left.k \frac{\partial T}{\partial n_{0}}\right|_{\Gamma}=0$

where $T_{0}$ is the initial temperature, $T_{k}$ is the known temperature, $T_{\infty}$ is the 
environmental temperature, $q_{0}$ is the prescribed heat flux, $h_{c}$ is the convection coefficient, $n_{0}$ is the unit outward normal to the boundary and $\Gamma$ represents the boundary.

The finite element method is utilized to deal with Eq. (1) and the discrete system equations are directly given as

$$
\mathbf{K}\{\mathbf{T}\}+\mathbf{N}\left\{\frac{\partial \mathbf{T}}{\partial t}\right\}=\left\{\mathbf{R}_{c}\right\}+\left\{\mathbf{R}_{q}\right\}
$$

where,

$$
\begin{aligned}
& \mathbf{K}=\int_{V} \mathbf{B}^{\mathrm{T}}\left[\begin{array}{ccc}
k_{x} & 0 & 0 \\
0 & k_{y} & 0 \\
0 & 0 & k_{z}
\end{array}\right] \mathbf{B} d V \\
& \mathbf{N}=\int_{V} \mathbf{H}^{\mathrm{T}} \mathbf{H} \rho c d V \\
& \left\{\mathbf{R}_{c}\right\}=\int_{\Omega} \mathbf{H}^{\mathrm{T}} h_{c}\left(T_{\infty}-T\right) d \Omega \\
& \left\{\mathbf{R}_{q}\right\}=\int_{\Omega} \mathbf{H}^{\mathrm{T}} q_{0} d \Omega
\end{aligned}
$$

where $\mathbf{K}$ is the conduction matrix, $\mathbf{N}$ is the thermal capacitance matrix, $\left\{\mathbf{R}_{c}\right\}$ and $\left\{\mathbf{R}_{q}\right\}$ are the thermal load vectors.

In this study, the backward difference technique is utilized for the time approximation, which can avoid the oscillation. Then, $\left\{\frac{\partial \mathbf{T}(t)}{\partial t}\right\}$ can be given by

$$
\left\{\frac{\partial \mathbf{T}(t)}{\partial t}\right\}=\frac{\{\mathbf{T}(t)\}-\{\mathbf{T}(t-\Delta t)\}}{\Delta t}+O(\Delta t)
$$

Substituting Eq. (11) into Eq. (6), the following equation can be obtained.

$$
\left(\mathbf{K}+\frac{\mathbf{N}_{T}}{\Delta t}\right)\{\mathbf{T}(t)\}=\left\{\mathbf{R}_{c}\right\}+\left\{\mathbf{R}_{q}\right\}+\left(\frac{\mathbf{N}_{T}}{\Delta t}\right)\{\mathbf{T}(t-\Delta t)\}
$$

Once the initial temperature field is given, the temperature field at arbitrary time $t$ can be obtained by solving Eq. (12) step by step. When nonlinearity is considered, 
Newton-Raphson iteration method is used to solve Eq. (12) until the convergence is reached in one time step. Usually, the increase of time step will lead to the loss of precision and it is easy to see that when time step is small or the scale of problem is large, the computational effort may become huge. Therefore, the following section will provide the CA procedures to fully improve the efficiency for the transient nonlinear heat transfer analysis.

\section{Combined approximations for nonlinear thermal analysis}

\subsection{Nonlinear thermal equations}

In typical transient nonlinear heat transfer analysis, the following system equation is solved in each time step repeatedly

$\mathbf{K}_{T} \delta \mathbf{T}=\delta \mathbf{R}$

where $\mathbf{K}_{T}$ is the temperature-dependent tangent matrix, $\delta \mathbf{T}$ is the vector of temperature due to the residual thermal load vector $\delta \mathbf{R}$. The temperature vector $\mathbf{T}$ is updated iteratively by recurrence relation

$\mathbf{T}^{i}=\mathbf{T}^{i-1}+\delta \mathbf{T}^{i}$

In solution procedures using the Newton-Raphson method, $\mathbf{K}_{T}, \delta \mathbf{R}, \delta \mathbf{T}$ and $\mathbf{T}$ are calculated repeatedly in each iteration cycle until the convergence is reached. $\mathbf{K}_{T}$ can be rewritten as

$\mathbf{K}_{T}=\mathbf{K}_{0}+\Delta \mathbf{K}_{0}$

Where $\mathbf{K}_{0}$ is the initial tangent matrix, $\Delta \mathbf{K}_{0}$ is the change of tangent matrix due to the change of temperature vector $\mathbf{T} . \delta \mathbf{R}$ is given as 


$$
\delta \mathbf{R}^{i}=\mathbf{R}_{0}-\mathbf{R}^{i}
$$

Where $\mathbf{R}_{0}$ is the external thermal load vector and $\mathbf{R}$ can be calculated by

$$
\mathbf{R}^{i}=\mathbf{K}_{T} \mathbf{T}^{i-1}
$$

Then the following system equation can be obtained

$$
\left(\mathbf{K}_{0}+\Delta \mathbf{K}_{0}\right) \delta \mathbf{T}^{i}=\left(\mathbf{R}_{0}-\mathbf{R}^{i}\right)
$$

In order to reduce the computational cost, Eq. (18) is efficiently and accurately solved by CA method instead of complete analysis. Usually, global approximations (the reduced basis method) and local approximations (series expansion) are combined in the CA procedures and this will be introduced in the following sections.

\subsection{Reduced basis expression}

The temperature vector $\delta \mathbf{T}$ can be approximated by linear combination of $s$ linearly-independent basis vectors. Then, $\delta \mathbf{T}$ can be approximated as

$$
\delta \mathbf{T}=x_{1} \delta \mathbf{T}_{1}+x_{2} \delta \mathbf{T}_{2}+\cdots x_{s} \delta \mathbf{T}_{s}=\delta \mathbf{T}_{B} \mathbf{x}
$$

where

$$
\begin{aligned}
& \delta \mathbf{T}_{B}=\delta \mathbf{T}_{1}, \cdots, \delta \mathbf{T}_{s} \\
& \mathbf{x}=\left\{\begin{array}{c}
x_{1} \\
\vdots \\
x_{s}
\end{array}\right\}
\end{aligned}
$$

$\mathbf{x}$ is a vector of coefficients to be determined. Substituting Eq. (19) into Eq. (18) and premultiplying by $\delta \mathbf{T}_{B}^{\mathrm{T}}$, the following equation can be obtained

$$
\delta \mathbf{T}_{B}^{\mathrm{T}}\left(\mathbf{K}_{0}+\Delta \mathbf{K}_{0}\right) \delta \mathbf{T}_{B} \mathbf{x}=\delta \mathbf{T}_{B}^{\mathrm{T}}\left(\mathbf{R}_{0}-\mathbf{R}\right)
$$

Introducing the following notation

$$
\mathbf{K}_{B}=\delta \mathbf{T}_{B}^{\mathrm{T}}\left(\mathbf{K}_{0}+\Delta \mathbf{K}_{0}\right) \delta \mathbf{T}_{B}
$$




$$
\mathbf{R}_{B}=\delta \mathbf{T}_{B}^{\mathrm{T}}\left(\mathbf{R}_{0}-\mathbf{R}\right)
$$

Then Eq. (22) can be rewritten as

$$
\mathbf{K}_{B} \mathbf{x}=\mathbf{R}_{B}
$$

Note that $s$ is much smaller than the number of system degrees of freedom $n$. The approximate temperature vector $\delta \mathbf{T}$ can be calculated by solving much smaller system equations $(s \times s)$ in Eq. (25), instead of solving the large $(n \times n)$ system equations in Eq. (18). Once $\mathbf{x}$ is obtained, the temperature vector $\delta \mathbf{T}$ can be easily evaluated by Eq. (19).

\subsection{Binomial series expansion}

A basic part of CA approach is the construction of basis vectors. In present work, terms of binomial series are selected as basis vectors, which have many advantages, such as accuracy, generality, efficiency and ease of implementation. The basis vectors $\delta \mathbf{T}_{1}, \delta \mathbf{T}_{2}, \cdots, \delta \mathbf{T}_{s}$ are determined by the following procedures.

Nonlinear Eq. (18) can be rearranged as

$\delta \mathbf{T}=\mathbf{K}_{0}^{-1}\left(\delta \mathbf{R}-\Delta \mathbf{K}_{0} \delta \mathbf{T}\right)$

Introducing notation $\mathrm{B}$,

$\mathbf{B}=\mathbf{K}_{0}^{-1} \Delta \mathbf{K}_{0}$

Substitute Eq. (27) into Eq. (26) and write Eq. (26) as the recurrence relation,

$\delta \mathbf{T}^{(j+1)}=\mathbf{K}_{0}^{-1} \delta \mathbf{R}-\mathbf{B} \delta \mathbf{T}^{(j)}$

Where, $\delta \mathbf{T}^{(j+1)}$ is the value of $\delta \mathbf{T}$ after $j$-th cycle. The initial value is given as

$\delta \mathbf{T}^{(1)}=\mathbf{K}_{0}^{-1} \delta \mathbf{R}$

Then the following relation can be obtained 
$\delta \mathbf{T}=\delta \mathbf{T}^{(1)}-\mathbf{B} \delta \mathbf{T}^{(1)}+\mathbf{B}^{2} \delta \mathbf{T}^{(j)}-\cdots+\mathbf{B}^{s-1} \delta \mathbf{T}^{(1)}$

The selected series of basis vectors can be calculated by

$$
\begin{aligned}
& \delta \mathbf{T}_{1}=\delta \mathbf{T}^{(1)}=\mathbf{K}_{0}^{-1} \delta \mathbf{R} \\
& \delta \mathbf{T}_{2}=-\mathbf{B} \delta \mathbf{T}_{1} \\
& \vdots \\
& \delta \mathbf{T}_{s}=-\mathbf{B} \delta \mathbf{T}_{s-1}
\end{aligned}
$$

Where $s$ is the number of basis vectors. Note that the decomposed form of $\mathbf{K}_{0}$ can be given as

$$
\mathbf{K}_{0}=\mathbf{U}^{T} \mathbf{U}
$$

Where $\mathbf{U}$ is an upper triangular matrix. Then the calculation of $\delta \mathbf{T}_{1}$ involves only forward and back substitutions and the calculation of $s$-th basis vector $\delta \mathbf{T}_{s}$ can be carried out by

$$
\mathbf{K}_{0} \delta \mathbf{T}_{s}=-\Delta \mathbf{K}_{0} \delta \mathbf{T}_{s-1}
$$

The forward substitution and backward substitution can be given as

$$
\mathbf{U}^{T} \delta \mathbf{T}_{0}=-\Delta \mathbf{K}_{0} \delta \mathbf{T}_{s-1}
$$

$$
\mathbf{U} \delta \mathbf{T}_{s}=\delta \mathbf{T}_{0}
$$

It can be seen that the calculation of each basis vector is very easy to implement and hence, the presented CA procedures can be simply carried out through a few revisions on existing finite element method codes. A brief description of overall analysis procedure using CA method is given in Fig. 1.

\section{Results and discussion}

Both two-dimensional and three-dimensional transient nonlinear heat transfer problems are examined to fully test the accuracy and efficiency of presented CA 
method. For comparison, complete analysis is also implemented by FEM in-house codes and the thermal equivalent energy is defined as

$$
E_{T}=\int_{V} \mathbf{B}^{\mathrm{T}}\left[\begin{array}{ccc}
k_{x} & 0 & 0 \\
0 & k_{y} & 0 \\
0 & 0 & k_{z}
\end{array}\right] \mathbf{B} d V
$$

The comparison of computational efficiency between CA method and complete analysis is carried out on the same computer of $\operatorname{Inter}^{\circledR}$ Pentium (R) D CPU $3.00 \mathrm{GHz}$.

\subsection{D case}

Transient nonlinear heat conduction analysis of a square plate is first presented in this section, which is subjected to mixed boundary conditions. Geometry and the boundary conditions are shown in Fig. 2. The related parameters used in the computation are taken as, $l_{1}=l_{2}=0.5 \mathrm{~m}, k_{x}, k_{y} k_{z}=(303.15+0.3 T) \mathrm{W} / \mathrm{m} \cdot \mathrm{K}, c=200 \mathrm{~J} / \mathrm{kg} \cdot \mathrm{K}$ and $\rho=500 \mathrm{~kg} / \mathrm{m}^{3}$. For the Dirichlet boundary $T_{k}=293.15 \mathrm{~K}$, for the Neumann boundary $q_{0}=-1000 \mathrm{~W} / \mathrm{m}^{2}$, and for the Robin boundary $h_{c}=100 \mathrm{~W} / \mathrm{m}^{2} \cdot \mathrm{K}, T_{\infty}=473.15 \mathrm{~K}$. Due to nonlinearity, the Newton-Raphson iteration method is utilized in both CA analysis and complete analysis. Different scales of meshes are studied in this example. The accuracy of CA method is examined using triangular meshes with 676 nodes and 1681 nodes, respectively. Fig. 3 shows the comparison of temperature at the middle point of the square plate using 676 nodes, when $t=100 \mathrm{~s}$, in which the CA solutions are obtained using different number of basic vectors. Fig. 4 plots the comparison of thermal equivalent energy using 1681 nodes, when $t=200 \mathrm{~s}$. It is clearly seen that solutions obtained by CA method agree well with those obtained by complete analysis, when using four or five basic vectors in this numerical example. Then, the 
computational effort of CA method using different number of basic vectors is compared in Fig. 5. Note that the computational effort $t_{r}$ includes the calculation of each basis vector, solving Eq. (19) and Eq. (25) in one iteration. It is observed that $t_{r}$ increases almost linearly when using different number of basic vectors. Generally, $t_{r}$ is much less than the computational effort that needed for the complete analysis in one iteration, especially when the scale of model is large. Furthermore, Fig. 6 plots the total computational effort of CA method and complete analysis for different scale of meshes, when the system has already reached its steady state. Four basic vectors are used in the comparison. It is shown that the computational effort of CA method is much smaller than that needed to carry out the complete analysis. For the analysis of transient nonlinear heat transfer problems, the advantage of CA method is more apparent when the number of degrees of freedom becomes large.

\section{2 $3 D$ case}

In this section, a 3D engine pedestal is studied as illustrated in Fig. 7. The engine pedestal has a central cylindrical hole in $z$-direction with radius $r_{1}=0.07 \mathrm{~m}$. In addition, the plate is subjected to the Neumann and Robin boundary conditions. Some computational parameters are given as $l_{1}=l_{2}=0.4 \mathrm{~m}, \quad r_{2}=0.12 \mathrm{~m}, \quad k_{x}, \quad k_{y}$ $k_{z}=(303.15+0.1 T) \mathrm{W} / \mathrm{m} \cdot \mathrm{K}, c=50 \mathrm{~J} / \mathrm{kg} \cdot \mathrm{K}$ and $\rho=300 \mathrm{~kg} / \mathrm{m}^{3}$. For the Neumann boundary $q_{0}=-6000 \mathrm{~W} / \mathrm{m}^{2}$, and for the Robin boundary $h_{c}=320 \mathrm{~W} / \mathrm{m}^{2} \cdot \mathrm{K}, T_{\infty}=573.15 \mathrm{~K}$. The problem domain is discretized into different scale of meshes. Then, the CA procedure and complete analysis are both implemented using these meshes.

In the $3 \mathrm{D}$ case, the accuracy of CA method is investigated using tetrahedron elements. 
For tetrahedron meshes with 752 nodes and 2395 nodes, thermal equivalent energy is calculated using different number of basic vectors. The obtained results are compared in Fig. 8 and Fig. 9, when $t=200 \mathrm{~s}$. In addition, Fig. 10 shows the comparison of temperature at point A using 6786 nodes, when $t=200 \mathrm{~s}$, in which the CA solutions are obtained using different number of basic vectors. It is clearly seen that when using three or more basic vectors, the CA method can achieve very high level of accuracy in this numerical example. The computational effort $t_{r}$ is also studied in the $3 \mathrm{D}$ case. Using meshes with 752 nodes and 2395 nodes, the value of $t_{r}$ is plotted in Fig. 11. As in the $2 \mathrm{D}$ case, $t_{r}$ changes linearly when using different basic vectors. Finally, the total computational effort of CA method and complete analysis are compared in Fig. 12, when the system has already reached its steady state. Four basic vectors are utilized in the CA analysis. It can be observed that the computational effort saving of CA procedures is significant, compared with complete analysis. Base on these results, we can come to the conclusion that the presented CA procedure can achieve high level of accuracy and efficiency, when solving transient nonlinear heat conduction problems.

\section{Conclusion}

In this work, an efficient Combined Approximations (CA) approach is presented for transient nonlinear heat conduction analysis. The solution procedure is straightforward and can be easily carried out using exiting FEM codes. Both 2D and 3D numerical examples are investigated to examine the validity of CA method when solving transient nonlinear heat transfer problems. From these studies, several 
conclusions can be made as follows:

1. The calculation of basic vectors is easy to implement. Compared with the complete analysis, it involves much less computational effort.

2. The CA method can achieve high level of accuracy by using a small number of basic vectors.

3. The computational effort saving of CA procedure becomes large when the number of degrees of freedom increases.

\section{Acknowledgements}

The support of National Science Foundation of China (11472101) and Priority Academic Program Development of Jiangsu Higher Education Institutions are gratefully acknowledged.

\section{References}

[1] M.T. Yang, K.H. Park, P.K. Banerjee, 2D and 3D transient heat conduction analysis by BEM via particular integrals, Comput. Methods Appl. Mech. Eng., 191 (2002) 1701-1722.

[2] M. Tanaka, T. Matsumoto, S. Takakuwa, Dual reciprocity BEM for time-stepping approach to the transient heat conduction problem in nonlinear materials, Comput. Methods Appl. Mech. Eng., 195 (2006) 4953-4961.

[3] J. Yang, X.L. Huang, Nonlinear transient response of functionally graded plates with general imperfections in thermal environments, Comput. Methods Appl. Mech. Eng., 196 (2007) 2619-2630.

[4] A. Khosravifard, M.R. Hematiyan, L. Marin, Nonlinear transient heat conduction analysis of functionally graded materials in the presence of heat sources using an improved meshless radial point interpolation method, Appl. Math. Modell., 35 (9) (2011) 4157-4174.

[5] A. Korzeń, D. Taler, Modeling of transient response of a plate fin and tube heat exchanger, Int. J. Therm. Sci., 92 (2015) 188-198.

[6] A. Matine, N. Boyard, G. Legrain, Y. Jarny, P. Cartraud, Transient heat conduction within periodic heterogeneous media: A space-time homogenization approach, Int. J. Therm. Sci., 92 (2015) 217-229.

[7] S.Z. Feng, X.Y. Cui, G.Y. Li, Thermo-mechanical analysis of composite pressure vessels using edge-based smoothed finite element method, Int. J. Comput. Methods, 11 (6) (2014).

[8] E. Li, Z.P. Zhang, Z.C. He, Smoothed finite element method with exact solutions in heat transfer 
problems, Int. J. Heat Mass Transf., 78 (2014) 1219-1231.

[9] I.V. Singh, A numerical solution of composite heat transfer problems using meshless method, Int. J. Heat Mass Transf., 47(10-11) (2004) 2123-2138.

[10] A. Singh, I.V. Singh, R. Prakash, Meshless element free Galerkin method for unsteady nonlinear heat transfer problems, Int. J. Heat Mass Transf., 50 (5-6) (2007) 1212-1219.

[11] M. Shibahara, S.N. Atluri, The meshless local Petrov-Galerkin method for the analysis of heat conduction due to a moving heat source, in welding, Int. J. Therm. Sci., 50 (6) (2011) 984-992.

[12] A. Khosravifard, M.R. Hematiyan, L. Marin, Nonlinear transient heat conduction analysis of functionally graded materials in the presence of heat sources using an improved meshless radial point interpolation method, Appl. Math. Modell., 35 (9) (2011) 4157-4174.

[13] S.C. Wu, G.R. Liu, H.O. Zhang, G.Y. Zhang, A node-based smoothed point interpolation method (NS-PIM) for thermoelastic problems with solution bounds, Int. J. Heat Mass Transf., 52 (5-6) (2009) 1464-1471.

[14] S.C. Wu, G.R. Liu, X.Y. Cui, T.T. Nguyen, G.Y. Zhang, An edge-based smoothed point interpolation method (ES-PIM) for heat transfer analysis of rapid manufacturing system, Int. J. Heat Mass Transf., 53 (9-10) (2010) 1938-1950.

[15] X.Y. Cui, S.Z. Feng, G.Y. Li, A cell-based smoothed radial point interpolation method (CS-RPIM) for heat transfer analysis, Eng. Anal. Boundary Elem., 40 (2014) 147-153.

[16] M. Mohammadi, M.R. Hematiyan, L. Marin, Boundary element analysis of nonlinear transient heat conduction problems involving non-homogenous and nonlinear heat sources using time-dependent fundamental solutions, Eng. Anal. Boundary Elem., 34(7) (2010) 655-665.

[17] U. Kirsch, Reduced basis approximations of structural displacements for optimal design, AIAA. J., 29 (1991) 1751-1758.

[18] U. Kirsch, Efficient reanalysis for topological optimization, Struct. Multidisc. Optim., 6 (1993) 143-150.

[19] U. Kirsch, Effective sensitivity analysis for structural optimization, Comput. Methods Appl. Mech. Eng., 117 (1994) 143-156.

[20] U. Kirsch, Efficient-accurate reanalysis for structural optimization., AIAA. J., 37 (1999) 1663-1669.

[21] U. Kirsch, Combined approximations - a general reanalysisapproach for structural optimization, Struct. Multidisc. Optim., 20 (2000) 97-106.

[22] U. Kirsch, A unified reanalysis approach for structural analysis, design and optimization, Struct. Multidisc. Optim., 25 (2003) 67-85.

[23] U. Kirsch, M. Bogomolni, Procedures for approximat eigenproblem reanalysis of structures, Int. J. Numer. Method Eng., 60 (2004) 1969-1998.

[24] U. Kirsch, M. Bogomolni, Error evaluation in approximat reanalysis of structures, Struct. Multidisc. Optim., 28 (2004) 77-86.

[25] U. Kirsch, M. Bogomolni, I. Sheinman, Nonlinear dynamic reanalysis of structures by combined approximations, Comput. Methods Appl. Mech. Eng., 195 (2006) 4420-4432.

[26] U. Kirsch, M. Bogomolni, Nonlinear and dynamic structura analysis using linear reanalysis, Comput. Struct., 85 (2007) 566-578.

[27] F. Rong et al., Structural modal reanalysis for topological modifications with extended Kirsch method, Comput. Methods Appl. Mech. Eng., 192 (2003) 697-707.

[28] M. Bogomolni, U. Kirsch, I. Sheinman, Efficient design-sensitivities of structures subjected to 
dynamic loading, Int. J. Solids Struct., 43 (2006) 5485-5500.

[29] O. Amir, U. Kirsch, I. Sheinman, Efficient nonlinear reanalysis of skeletal structures using combined approximations, Int. J. Numer. Method Eng., 73 (2008) 1328-1346.

[30] H. Wang, E.Y. Li, G.Y. li, A parallel reanalysis method based on approximate inverse matrix for complex engineering problems, J. Mech. Design, 135 (8) (2013).

[31] G. Huang, H. Wang, G.Y. li, A reanalysis method for local modification and the application in large-scale problems, Struct. Multidisc. Optim., 49 (2014) 915-930.

[32] H. Wang, Y. Zeng, E.Y. Li, "Seen Is Solution”(SIS), a CAD/CAE integrated parallel reanalysis design system, Comput. Methods Appl. Mech. Eng., In press (2015). 


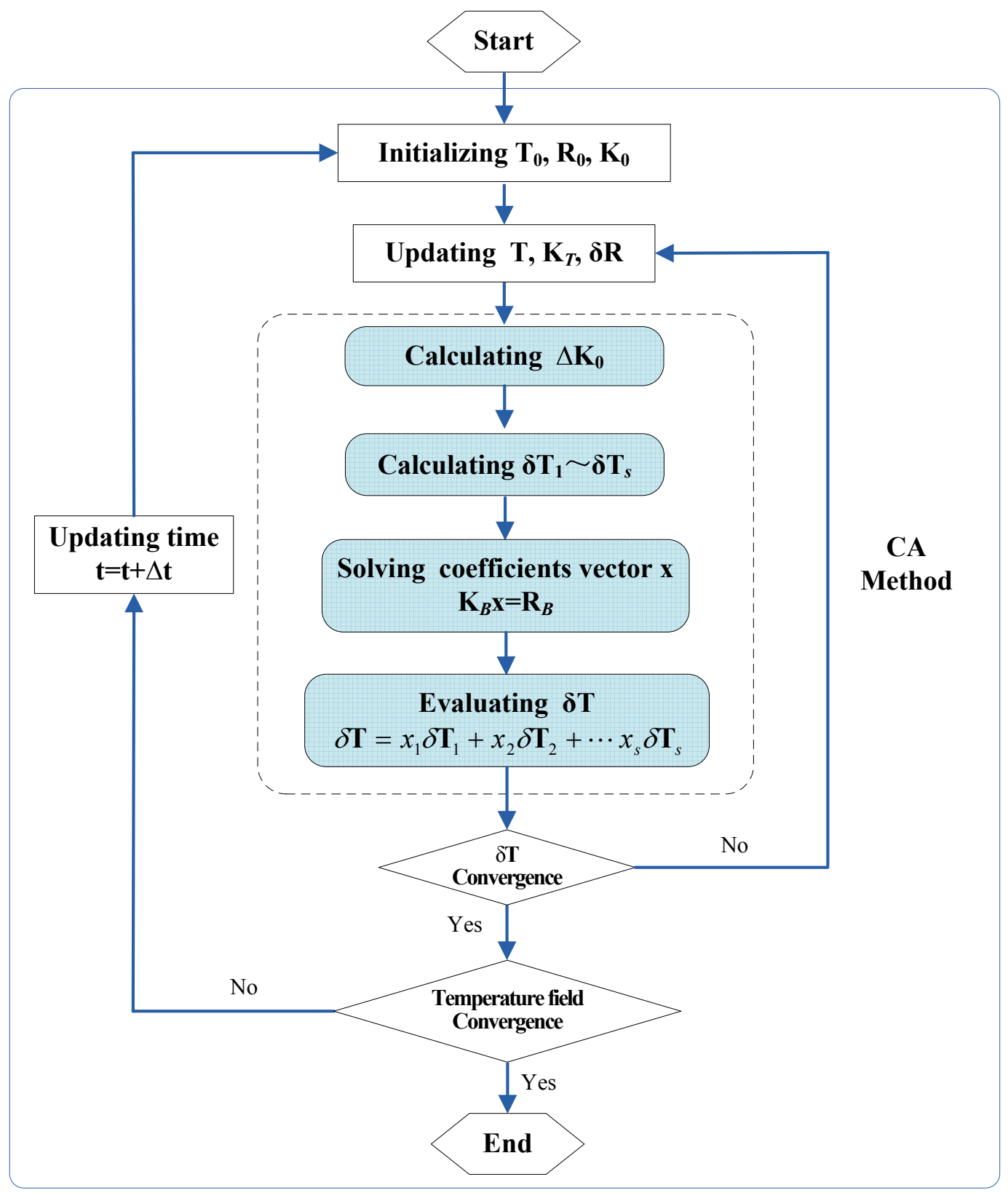

Fig. 1. Brief description of overall analysis procedures 


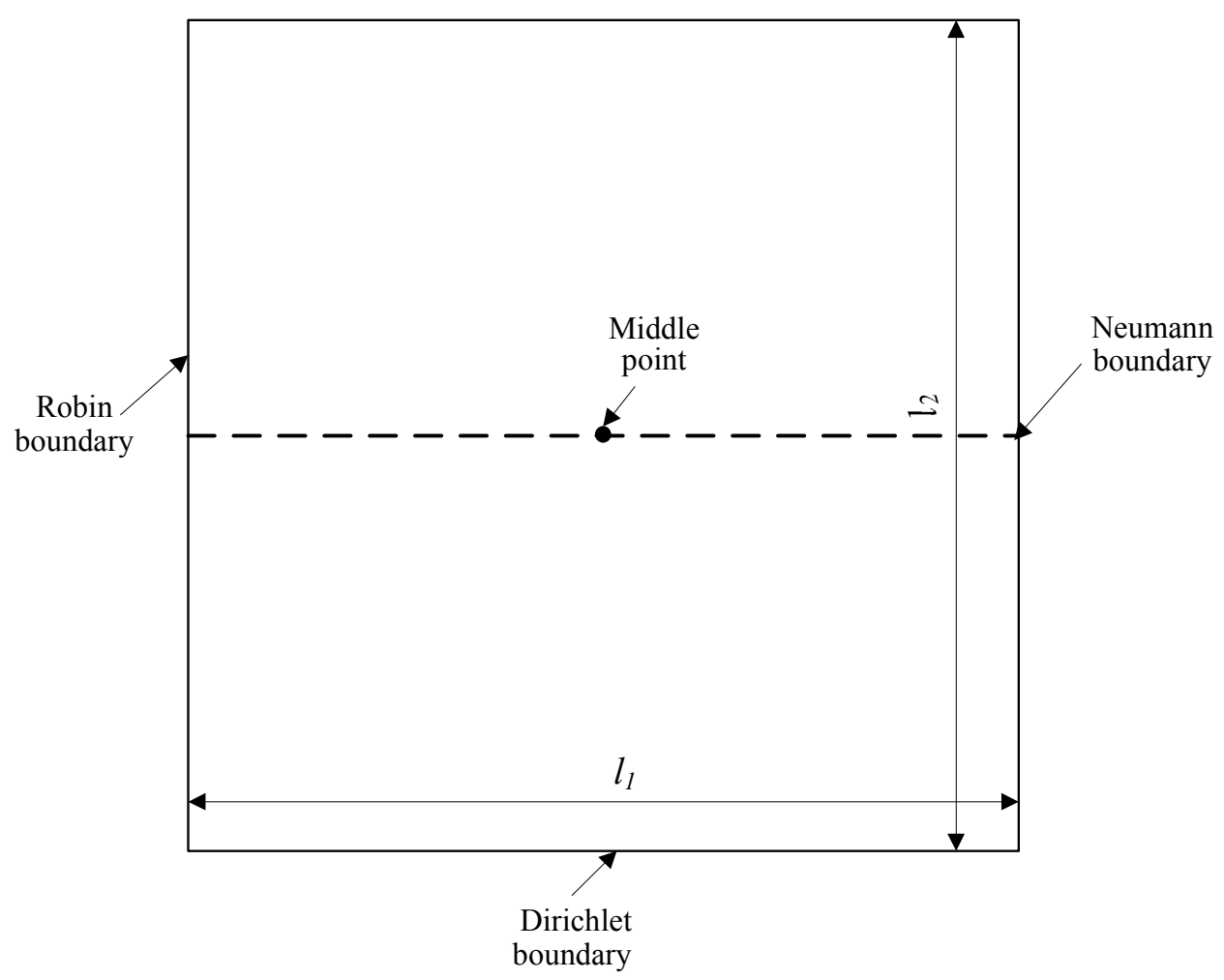

Fig. 2. The square plate with mixed boundary conditions 


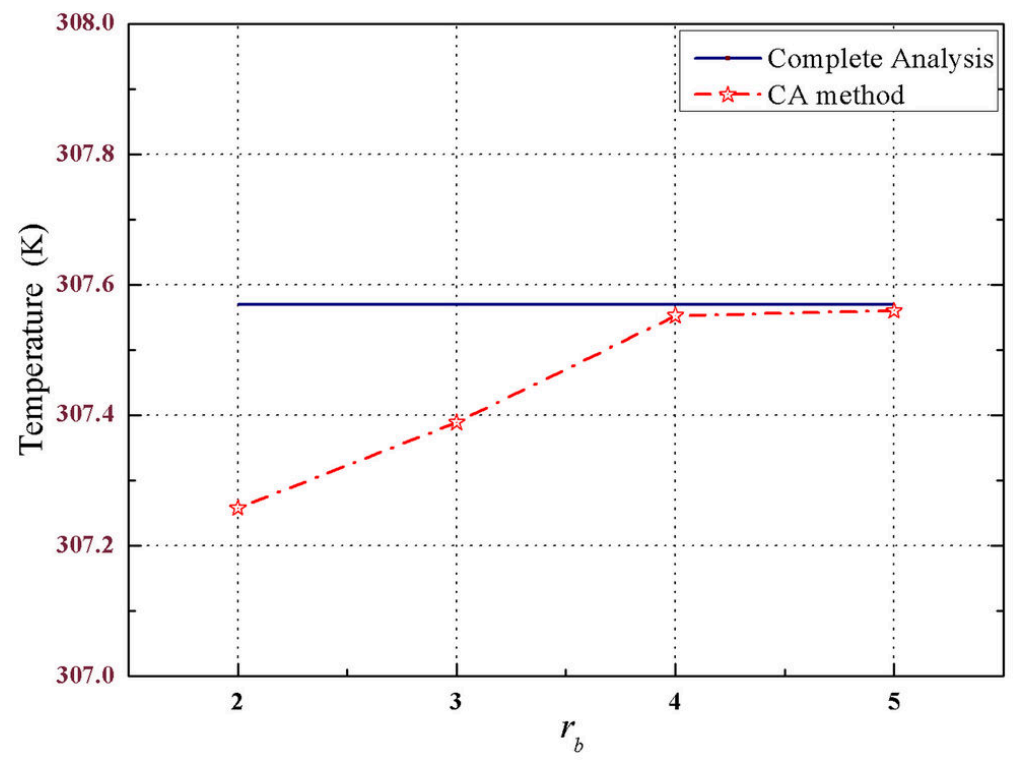

Fig. 3. The comparison of temperature at the middle point of the square plate using different number of basic vectors (676 nodes mesh) 


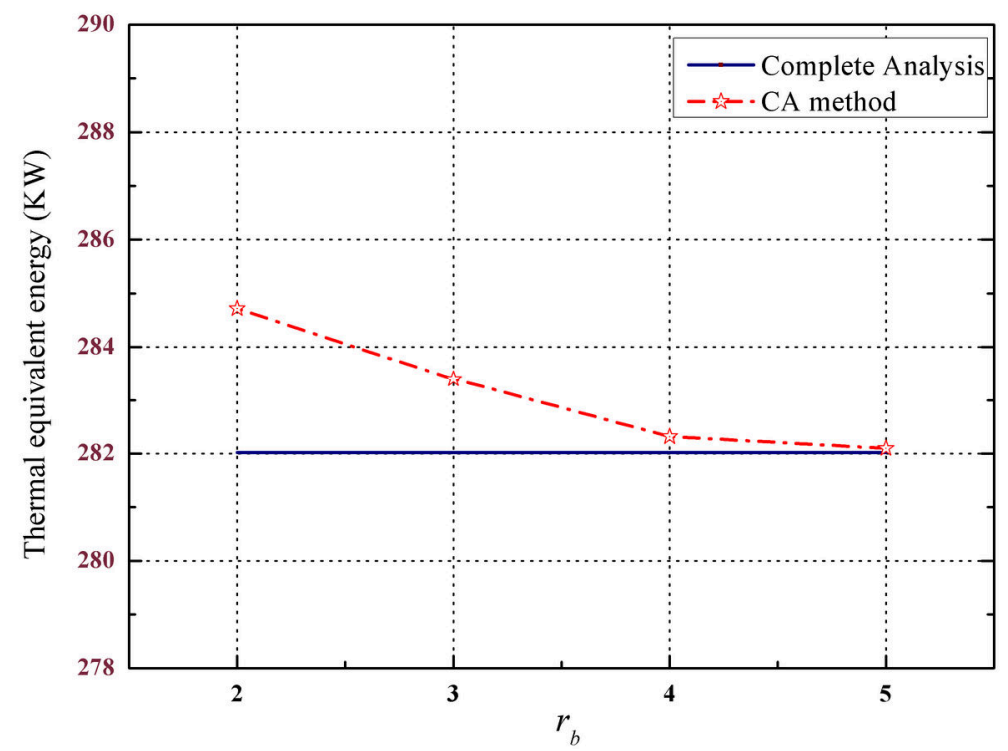

Fig. 4. The comparison of thermal equivalent energy using different number of basic vectors (1681 nodes mesh) 


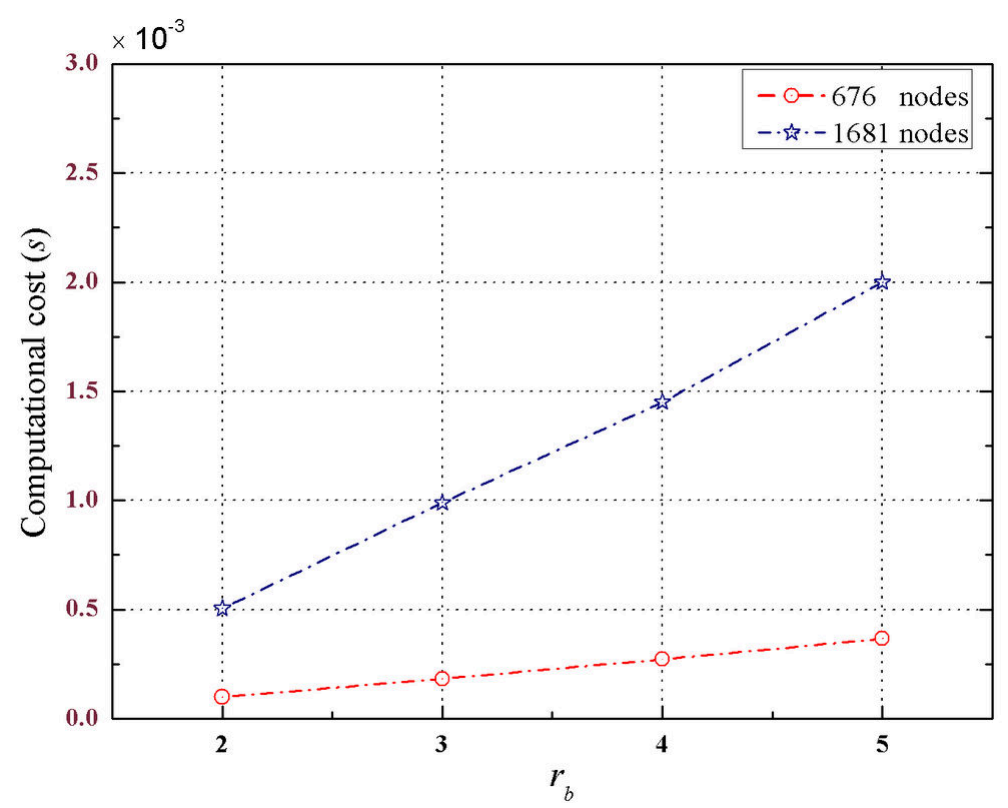

Fig. 5. The computational effort $t_{r}$ for different number of basic vectors 


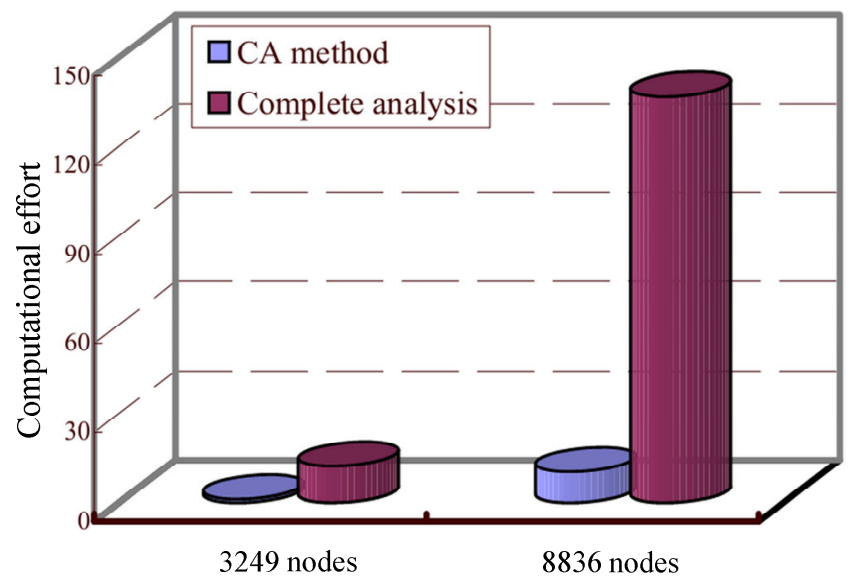

Fig. 6. The comparison of computational effort for different meshes 


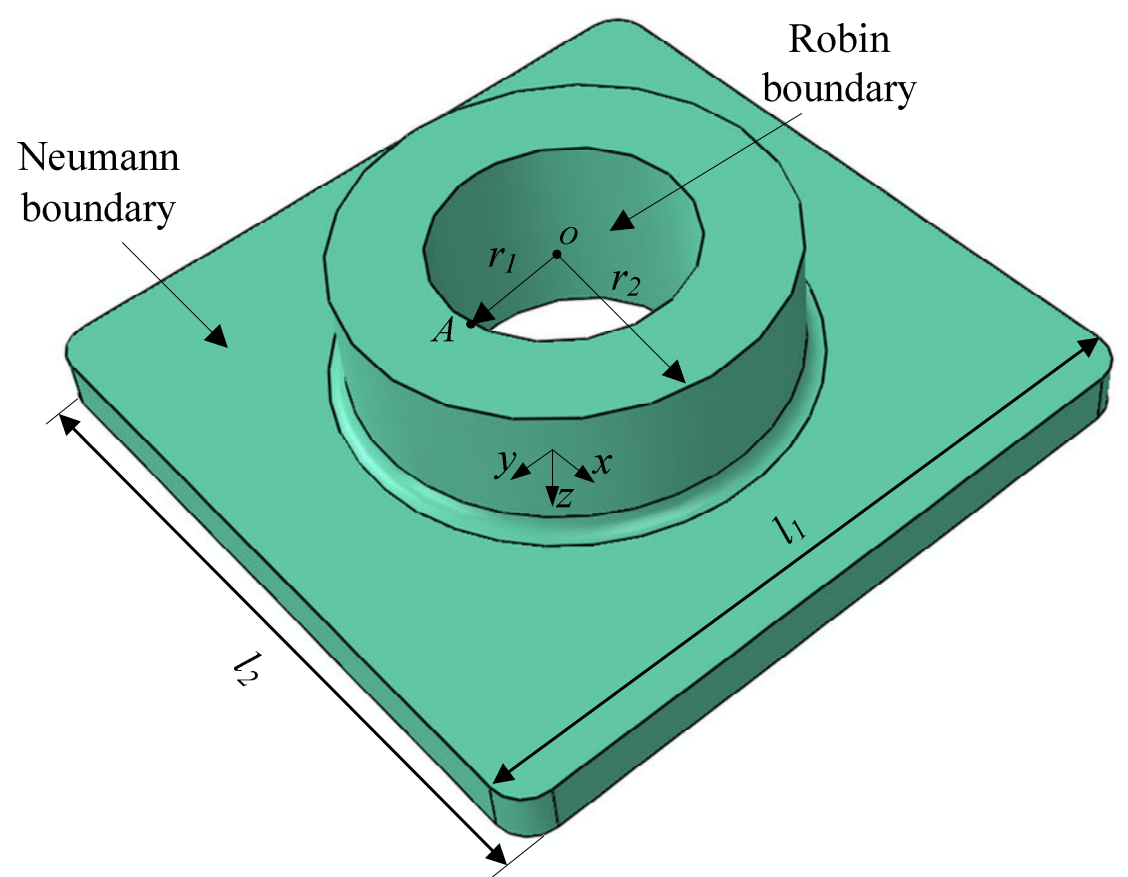

Fig. 7. Schematic of engine pedestal with mixed boundary conditions 


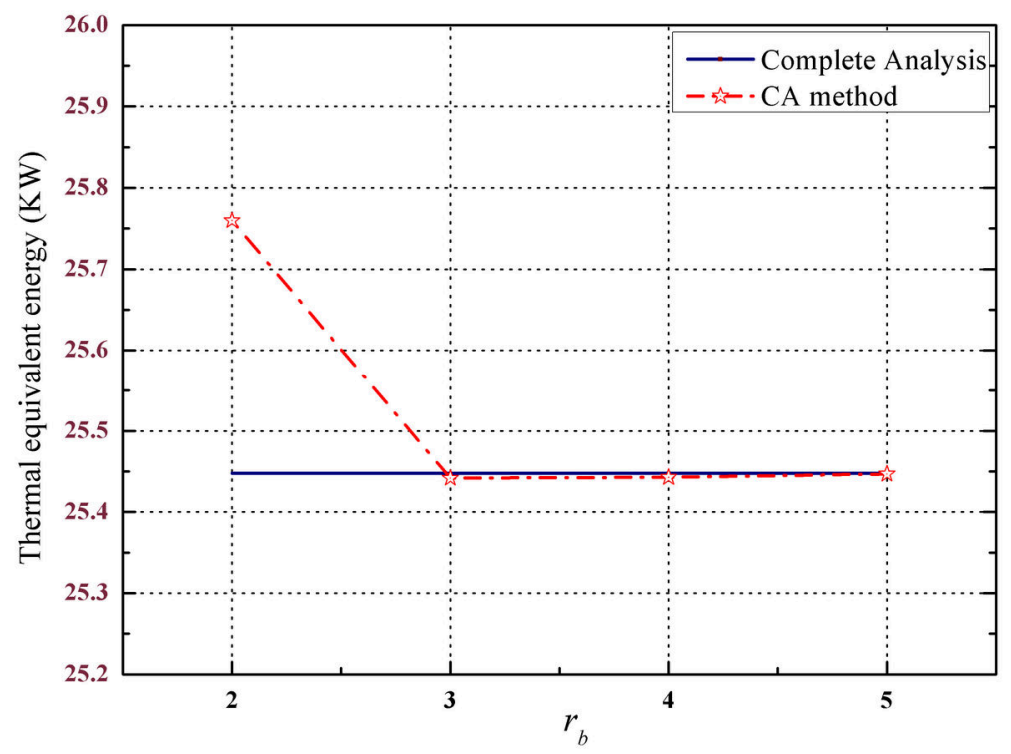

Fig. 8. The comparison of thermal equivalent energy using different number of basic vectors (752 nodes mesh) 


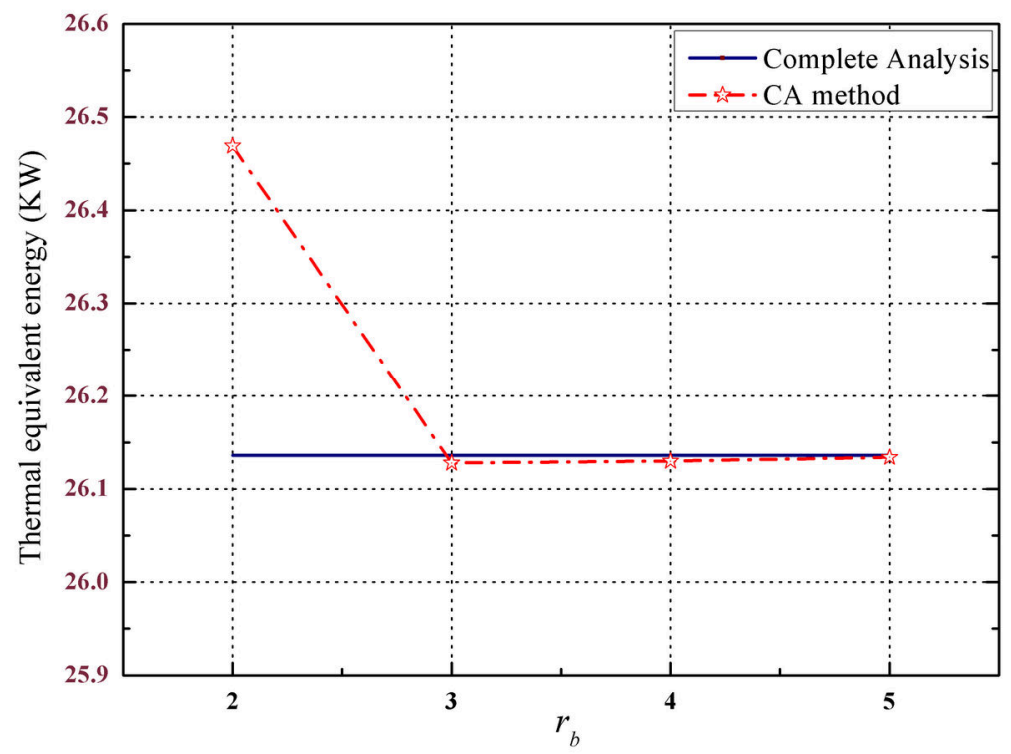

Fig. 9. The comparison of thermal equivalent energy using different number of basic vectors (2395 nodes mesh) 


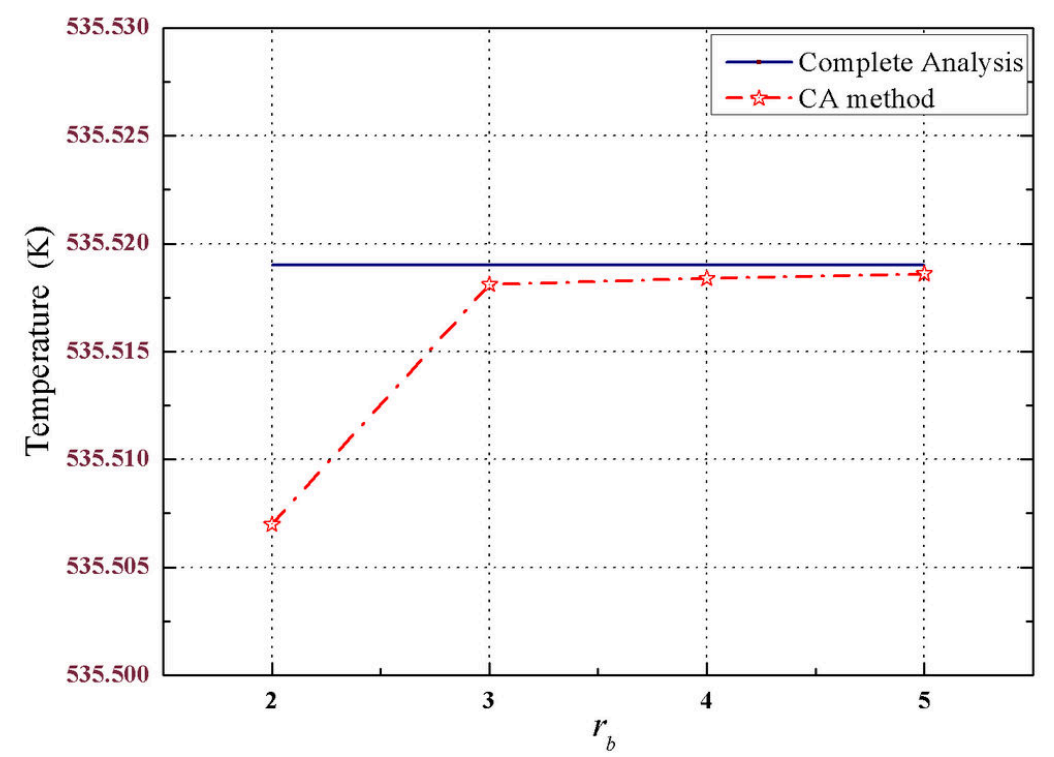

Fig. 10. The comparison of temperature at point $A(0,0.07,-0.08)$ using different number of basic vectors (6786 nodes mesh) 


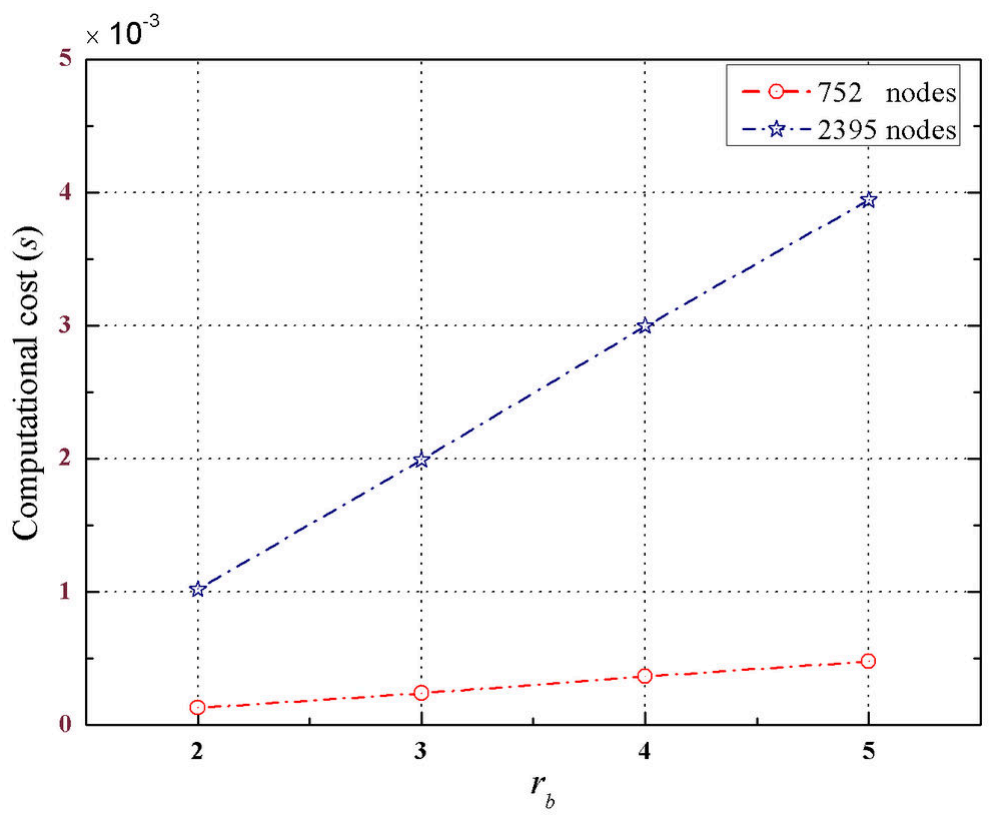

Fig. 11. The computational effort $t_{r}$ for different number of basic vectors. 


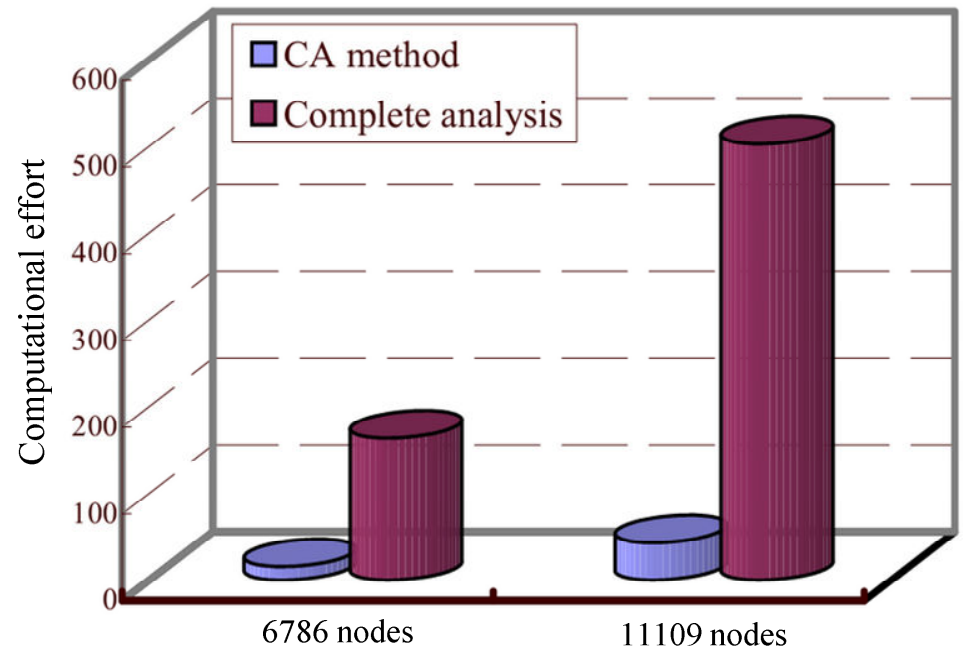

Fig. 12. The comparison of computational effort for different scale of meshes 